\title{
Bound states of a spin- $1 / 2$ charged particle in a magnetic flux tube
}

\author{
R. M. Cavalcanti* ${ }^{* \ddagger}$ and C. A. A. de Carvalho ${ }^{\dagger \S}$ \\ ${ }^{\ddagger}$ Institute for Theoretical Physics, University of California, Santa Barbara, CA 93106-4030, USA \\ $\S$ Instituto de Física, Universidade Federal do Rio de Janeiro, Caixa Postal 68528, Rio de Janeiro, \\ RJ 21945-970, Brasil
}

\begin{abstract}
We study a spin-1/2 charged particle with gyromagnetic factor $g>2$ moving in a plane threaded by a magnetic flux tube. We prove that, if the magnetic field (i) has radial symmetry, (ii) has compact support and (iii) does not change sign, there is at least one bound state for each angular momentum $\ell$ in the interval $[0, v]$, where $v$ (assumed positive) is the total magnetic flux in units of the quantum of flux.
\end{abstract}

PACS numbers: 03.65.-w, 03.65.Ge

*E-mail: rmc@itp.ucsb.edu

†E-mail: aragao@if.ufrj.br 
Let us consider a spin-1/2 particle with charge $e$ and gyromagnetic factor $g$, moving in a plane threaded by a magnetic field. Its dynamics is governed by the Pauli Hamiltonian, which, when specialized to the case where the magnetic field points in the $z$ direction, can be written as $\left[2 m=\hbar=c=1 ; \sigma_{z}=\operatorname{diag}(1,-1)\right]$

$$
H=(-i \nabla-e \mathbf{A})^{2}-\frac{1}{2} g e B \sigma_{z} \equiv \operatorname{diag}\left(H_{\uparrow}, H_{\downarrow}\right) .
$$

For $g=2$ - the value which emerges naturally when one derives the Pauli Hamiltonian as the non-relativistic limit of the Dirac Hamiltonian - , it is possible to show [1 3 ] that the above Hamiltonian has zero-energy normalizable' eigenstates if the total magnetic flux $\Phi \equiv \int B(\mathbf{x}) d^{2} x$ is larger than $\Phi_{0}=2 \pi /|e|$ (in general, there are $N$ such states if $N \Phi_{0}<$ $\left.\Phi \leq(N+1) \Phi_{0}\right)$.

On the other hand, the experimental value of the electron $g$-factor is not 2, but 2.0023 (this "anomaly" can be understood as a result of QED radiative corrections [4]). This has motivated some investigations of the case $g>2$ in the last few years. In this case, qualitatively new phenomena occur: (a) an arbitrarily small (but nonzero) magnetic flux $\Phi$ can create a negative-energy state [5], and (b) even if $\Phi=0$, bound states can appear if the magnetic field is strong enough [8].

A very important limitation of (a) is that it has been proved only for a few types of magnetic field profiles, and so it is not known to what extent it is a general result. The purpose of this paper is to partially fill this gap. We shall prove that, if $g>2$ and the magnetic field satisfies the following conditions: (i) it has radial symmetry, (ii) it has compact support (and so the flux tube has a well defined radius $\mathrm{R}$ ) and (iii) it does not change sign (without loss of generality, we shall suppose that $e B(r) \geq 0$ ), then $H_{\uparrow}$ has at least $[v]+1$ eigenstates with negative energy, where $v \equiv \Phi / \Phi_{0}$ and $[v]$ denotes its integer part (i.e., $v=[v]+\epsilon, 0 \leq \epsilon<1$ ). 2 In order to prove this assertion, we first note that, because of (i), we can choose the vector potential in the symmetric gauge, $\mathbf{A}=A_{\varphi}(r) \mathbf{e}_{\varphi}$, in terms of which the magnetic field is written as

$$
B(r)=\frac{1}{r} \frac{d}{d r}\left[r A_{\varphi}(r)\right]
$$

In this gauge, the time-independent Schrödinger equation is separable in polar coordinates $(r, \varphi)$, and one can perform a partial-wave decomposition.

Now, let us consider the following variational wave functions:

$$
\psi_{\ell}(r, \varphi)=\mathrm{e}^{i \ell \varphi} \times \begin{cases}a_{\ell} r^{\ell} \mathrm{e}^{-e \phi(r)-\alpha r} & \text { for } 0<r<R \\ b_{\ell} K_{|\ell-v|}(\mu r) \quad(\mu>0) & \text { for } r>R\end{cases}
$$

where $\phi(r) \equiv \int_{0}^{r} A_{\varphi}\left(r^{\prime}\right) d r^{\prime}$ and $K_{\nu}(z)$ is the modified Bessel function [9]. The coefficients $a_{\ell}$ and $b_{\ell}$ are chosen in such a way that $\psi_{\ell}(r, \varphi)$ is continuous at $r=R$ and $\left\langle\psi_{\ell} \mid \psi_{\ell}\right\rangle=1$.

\footnotetext{
${ }^{1}$ Such states are not normalizable if the magnetic field has a $\delta$-function singularity [6, [].

${ }^{2}$ Here, and in what follows, we are assuming that $v>0 . v=0$ corresponds to the absence of a magnetic field, in which case there are no bound states (instead of $[v]+1$ ).
} 
The latter condition can only be fulfilled if $\ell \geq 0$, in order that $\left|\psi_{\ell}\right|^{2}$ be integrable at the origin (integrability at infinity is ensured by the positivity of $\mu$ ). We shall also impose that $\partial_{r} \psi_{\ell}(r, \varphi)$ be continuous at $r=R$; together with the continuity of $\psi_{\ell}(r, \varphi)$, this implies that $\mu$ must satisfy the following equation:

$$
\frac{\mu R K_{|\ell-v|}^{\prime}(\mu R)}{K_{|\ell-v|}(\mu R)}=-v+\ell-\alpha R
$$

where we have used the identity

$$
\phi^{\prime}(R)=A_{\varphi}(R)=\frac{1}{R} \int_{0}^{R} B(r) r d r=\frac{v}{e R} .
$$

Since the l.h.s. of (1) tends to $-|\ell-v|$ when $\mu \rightarrow 0^{+}$, and it behaves asymptotically as $-\mu R$ when $\mu \rightarrow \infty$ [9], that equation will have a positive root if $\ell \leq v$ and $\alpha>0$.

We have thus obtained $[v]+1$ normalizable functions $\psi_{\ell}(r, \varphi)(\ell=0,1, \ldots,[v])$ which, together with $\partial_{r} \psi_{\ell}(r, \varphi)$, are continuous at $r=R$. Now, we shall show that, for each of these functions, it is possible to find a positive $\alpha$ such that $\left\langle\psi_{\ell}\left|H_{\uparrow}\right| \psi_{\ell}\right\rangle<0$. It then follows from the variational principle that there exists (at least) one negative energy eigenstate of $H_{\uparrow}$ with angular momentum $\ell$. In fact, a direct calculation shows that

$$
\begin{aligned}
\left\langle\psi_{\ell}\left|H_{\uparrow}\right| \psi_{\ell}\right\rangle= & 2 \pi\left|a_{\ell}\right|^{2} \int_{0}^{R}\left[\frac{(2 \ell+1) \alpha}{r}-2 \alpha e A_{\varphi}(r)-\alpha^{2}-\frac{1}{2}(g-2) e B(r)\right] r^{2 \ell} \mathrm{e}^{-2 e \phi(r)-2 \alpha r} r d r \\
& -2 \pi\left|b_{\ell}\right|^{2} \mu^{2} \int_{R}^{\infty} K_{|\ell-v|}^{2}(\mu r) r d r \\
\leq & 2 \pi\left|a_{\ell}\right|^{2} \int_{0}^{R}\left[\frac{(2 \ell+1) \alpha}{r}-\frac{1}{2}(g-2) e B(r)\right] r^{2 \ell} \mathrm{e}^{-2 e \phi(r)-2 \alpha r} r d r .
\end{aligned}
$$

Our goal will be accomplished if we choose a positive $\alpha$ satisfying

$$
\alpha<\frac{(g-2) e \int_{0}^{R} B(r) r^{2 \ell+1} \mathrm{e}^{-2 e \phi(r)-2 \alpha r} d r}{2(2 \ell+1) \int_{0}^{R} r^{2 \ell} \mathrm{e}^{-2 e \phi(r)-2 \alpha r} d r} .
$$

That such a choice is possible follows from the fact that the r.h.s. of the above inequality is

a continuous function of $\alpha$, and (if $v>0$ ) it tends to a positive number when $\alpha \rightarrow 0$. This completes the proof.

Finally, we make a couple of remarks: (i) an "extra" bound state (with $\ell>v$ ) can show up, provided $\ell-v<\alpha R / 2$ (in this case too one can prove that Eq. (州) has a positive root); (ii) our proof breaks down if $B$ has a $\delta$-function singularity at the origin; this case requires a special treatment (see discussion in Refs. [5, 6]), and will not be considered here.

\section{ACKNOWLEDGMENTS}

We thank Eduardo Souza Fraga for useful conversations. This work was finantially supported by the Conselho Nacional de Desenvolvimento Científico e Tecnológico (CNPq) and, in part, by the National Science Foundation under Grant No. PHY94-07194. 


\section{REFERENCES}

[1] Aharonov Y and Casher A 1979 Phys. Rev. A 19 2461-2

[2] Jackiw R 1984 Phys. Rev. D 29 2375-7

[3] Musto R, O’Raifeartaigh L and Wipf A 1986 Phys. Lett. B 175 433-8

[4] Schwinger J 1948 Phys. Rev. 73 416; 1949 Phys. Rev. 76 790-817; 1951 Phys. Rev. 82 664-79

[5] Bordag M and Voropaev S 1993 J. Phys. A: Math. Gen. 26 7637-49

[6] Moroz A 1996 Phys. Rev. A 53 669-94

[7] Cavalcanti R M, Fraga E S and de Carvalho C A A 1997 Phys. Rev. B 56 9243-6

[8] Bentosela F, Exner P and Zagrebnov V A 1998 J. Phys. A: Math. Gen. 31 L305-11

[9] Abramowitz M and Stegun I A (ed) 1965 Handbook of Mathematical Functions (New York: Dover) 